\title{
SEIS NOVAS LARVAS DO GÊNERO MICRATHYRIA KIRBY E NOTAS SOBRE A DISTRIBUIC̣ÃO NO BRASIL (ODONATA, LIBELLULIDAE)
}

\author{
Cristiane Vieira de Assis ${ }^{1}$ \\ Janira Martins Costa ${ }^{1}$
}

\begin{abstract}
Six NEW larvaE OF genus Micrathyrla Kirby and NOtes on DistribUtion in BRAZIL.(Odonata, LibellulidaE). Six new Micralhyria Kirby, 1889 larvae from Brazil are described and compared to those described by SANTOS (1968, 1972, 1978) and NEEDHAM (1943). The distribution of the species is studied based on material deposited in colection of Museu Nacional, Universidade Federal do Rio de Janeiro (MN-UFRJ).

KEY WORDS. Odonata, Libellulidae, Micrathyria, larvae, taxonomy
\end{abstract}

O gênero Micrathyria Kirby, 1889 inclui 36 espécies na América do Sul, das quais aproximadamente $80 \%$ têm registro no Brasil (DAVIES \& TOBIN 1985; RODRIGUES CAPITULO 1988; WESTFALl 1992).

Das 29 espécies registradas no Brasil, somente quatro têm suas larvas conheidas: M. artemis Ris, 1911, M. atra Martin, 1897, M. didyma Selys, 1857 e M. hypodidyma Calvert, 1906, segundo SANTOS (1988).

Neste trabalho as larvas de M. borgmeieri Santos, 1947, M. hesperis Ris, 1911, M. mengeri Ris, 1919, M. ocellata Martin, 1897, M. pirassunungae Santos, 1953 e M. stawiarskii Santos, 1953 são descritas pela primeira vez, comparando-as com as exúvias do último ínstar de $M$. artemis, $M$. atra, $M$. hypodidyma e com a descrição de $M$. didyma.

Segundo SANTOS et al. (1988) as larvas de Micrathyria até então conhecidas, possuem uma importância ecológica e econômica por representarem vorazes predadores de alevinos, e pela fácil adaptação em tanques de criação de peixes e em ambientes lênticos.

\section{Micrathyria borgmeieri Santos, 1947}

Figs 1-5

Larva pequena, de coloração ocrácea.

Cabeça tão larga quanto o tórax; olhos compostos arredondados lateralmente atingindo $2 / 3$ do comprimento médio-dorsal da cabeça; antenas com sete segmentos, sendo o penúltimo, mais escurecido que os demais; mandíbulas com dentes incisivos obtusos, dentes molares de forma irregular; fórmula mandibular

1) Departamento de Entomologia, Museu Nacional, universidade Federal do Rio de Janeiro, Quinta da Boa Vista, 20940-040 Rio de Janeiro, Rio de Janeiro, Brasil. Bolsista do CNPq. 


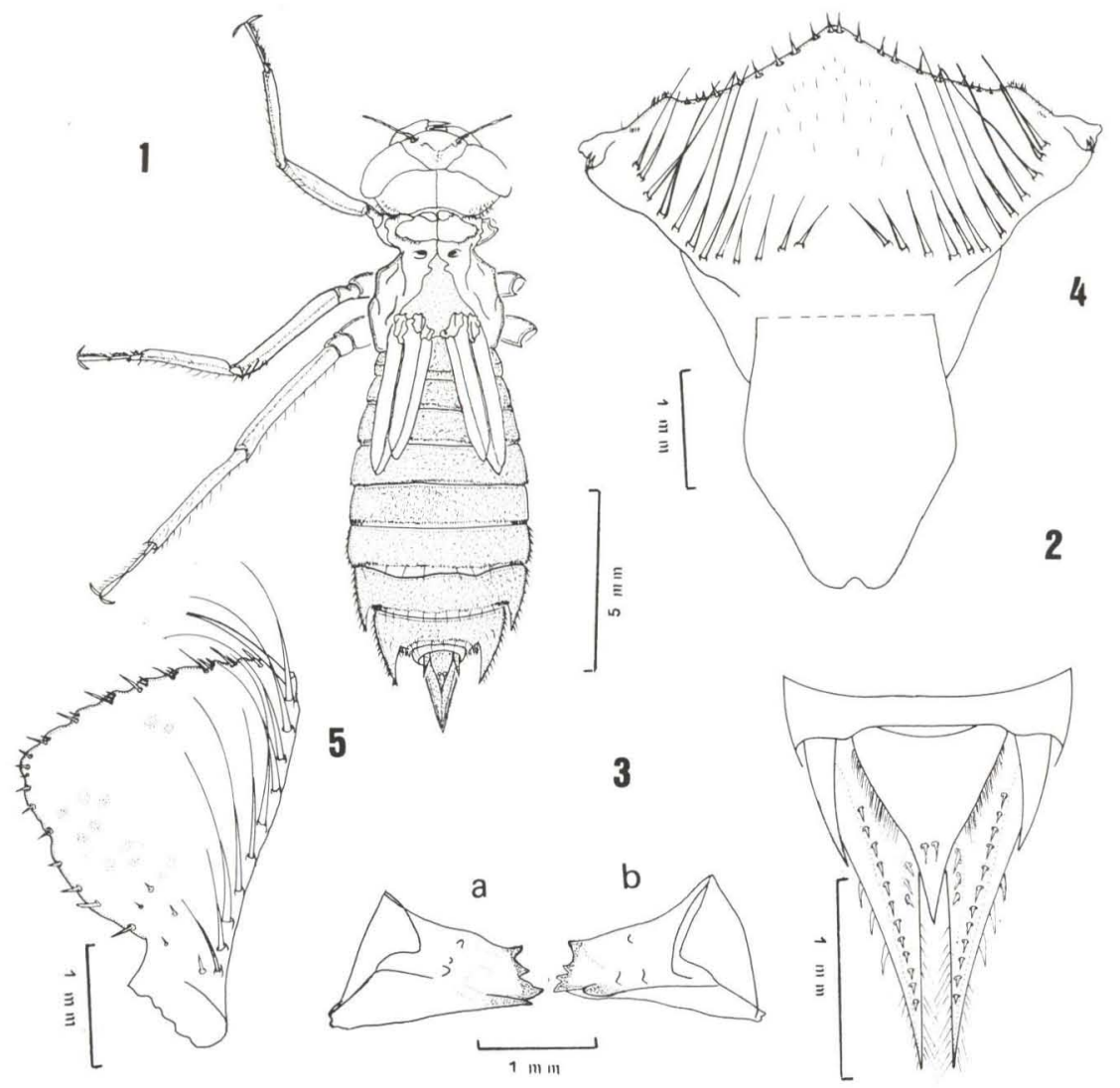

Figs 1-5. Micrathyria borgmeieri. (1) Exúvia de último ínstar; (2) apêndices abdominais; (3) mandibulas (a - esquerda, b - direita); (4) pré-mento; (5) palpo labial direito.

(de acordo com WATSON, 1956) $\frac{L}{R} \frac{1234}{1234} \frac{0}{y} \frac{a b}{a b d}$.

Lábio largo, quando dobrado, alcançando o nível do segundo par de pernas. Pré-mento com um par de 12 a 13 setas pré-mentais; margem distal com 11 setas espiniformes de cada lado e duas apicais; superfície interna com uma série de 23 pequenas cerdas transparentes; dois pequenos e robustos espinhos em cada lado da margem lateral, na conexão com os palpos labiais. Palpo labial largo, com pequenas máculas castanho-escuras; 10 setas e uma setela em cada palpo, além da garra móvel; parte basal com quatro pequenas setas espiniformes; margem distal com nove crenulações suaves, cada uma suportando de zero a três espinhos crenulares; número de espinhos em cada crenulação, partindo da garra móvel: 0 , $1,2,3,3,3,3,2,2$; margem interna com 12 setas irregularmente distribuídas; margem externa lisa.

Protórax com disco dorsal bordeado por cerdas. Tecas alares alcançando o 
nível do V segmento abdominal. Pernas longas armadas de fileiras de espinhos e cerdas.

Abdome ovalado, mais alargardo no VI segmento; margem externa provida de pequenos espinhos concentrados à partir do VII segmento (inclusive nos espinhos laterais VIII e IX); superfície dorsal com longos pêlos dispersos nas margens posteriores dos segmentos VII, VIII e IX; ausência de espinhos dorsais; espinhos laterais presentes nos segmentos VIII e IX; espinho do VIII segmento reto e estendendo-se até $2 / 3$ do comprimento médio-dorsal do IX segmento; espinho do IX segmento levemente curvado e duas vezes maior que o comprimento médio-dorsal do IX segmento, alcançando a extremidade do epiprocto. Epiprocto largo na base e fortemente afilado na extremidade; guarnecido por dois espinhos dispostos paralelamente em seu terço posterior. Paraprocto mais de duas vezes maior que o epiprocto e levemente curvado para baixo distalmente. Cerco alcançando aproximadamente $2 / 3$ do comprimento do epiprocto, levemente curvado para baixo distalmente. Todos providos de espinhos e pêlos nas extremidades.

Material examinado. BRASIL, Rio de Janeiro: Rio de Janeiro (Reserva Florestal do Tinguá), 23.II. 1972 (emergência 9.III. 1972), um macho, N.D. Santos leg., (Recreio dos Bandeirantes, Bosque da Barra) área aberta, 20.IV.1991, cinco machos, M.F. Napoli \& C.F. Bizerril leg., área de tabual, 18.V.1991, nove machos e duas fêmeas, M.F. Napoli \& C.F. Bizerril leg. , área aberta, 18.V.1991, dois machos, M.F. Napoli \& C.F. Bizerril leg.; depositado no MN-UFRJ.

Medidas (em milímetros). Comprimento total 16,95; comprimento da cabeça 2,85; largura máxima da cabeça 4,5; comprimento dos olhos 1,44; largura máxima dos olhos 1,68; comprimento das antenas 2,32; comprimento relativo dos antenômeros 20, 26, 37, 33, 37, 49, 30; comprimento do pré-mento 3,56; largura basal 1,38; largura máxima 2,92; comprimento do palpo labial 2,51; teca alar anterior 4,0; teca alar posterior 4,16; F1, F2, F3 (F=fêmur) 2,08, 2,72, 3,84; T1, T2, T3 ( $\mathrm{T}=$ tíbia) 2,48, 2,96, 4,0; comprimento do abdome 9,15; largura máxima 3,96; comprimento do espinho lateral VIII segmento 0,80 ; largura basal 0,23 ; comprimento do espinho lateral IX segmento 1,23 ; largura basal 0,42 ; epiprocto 0,96 ; paraprocto 2,07 ; cerco 0,75 .

Distribuição. BRAsIL, Espírito Santo: Jacareípe; Rio de Janeiro: Tinguá, Recreio dos Bandeirantes; Paraná:?.

\section{Micrathyria hesperis Ris, 1911}

Figs 6-10

Larva de tamanho pouco menor que a precedente, de coloração castanha.

Cabeça similar à da espécie anteriormente descrita; fórmula mandibular $L \underline{1234} 0$ abd $\bar{R} \overline{1234} \overline{0} \frac{a b d}{\text {. }}$

Lábio largo, quando dobrado alcançando o nível do segundo par de pernas. Pré-mento com um par de 11 setas pré-mentais; margem distal com seis setas espiniformes de cada lado e duas apicais; superfície interna com uma série de 28 


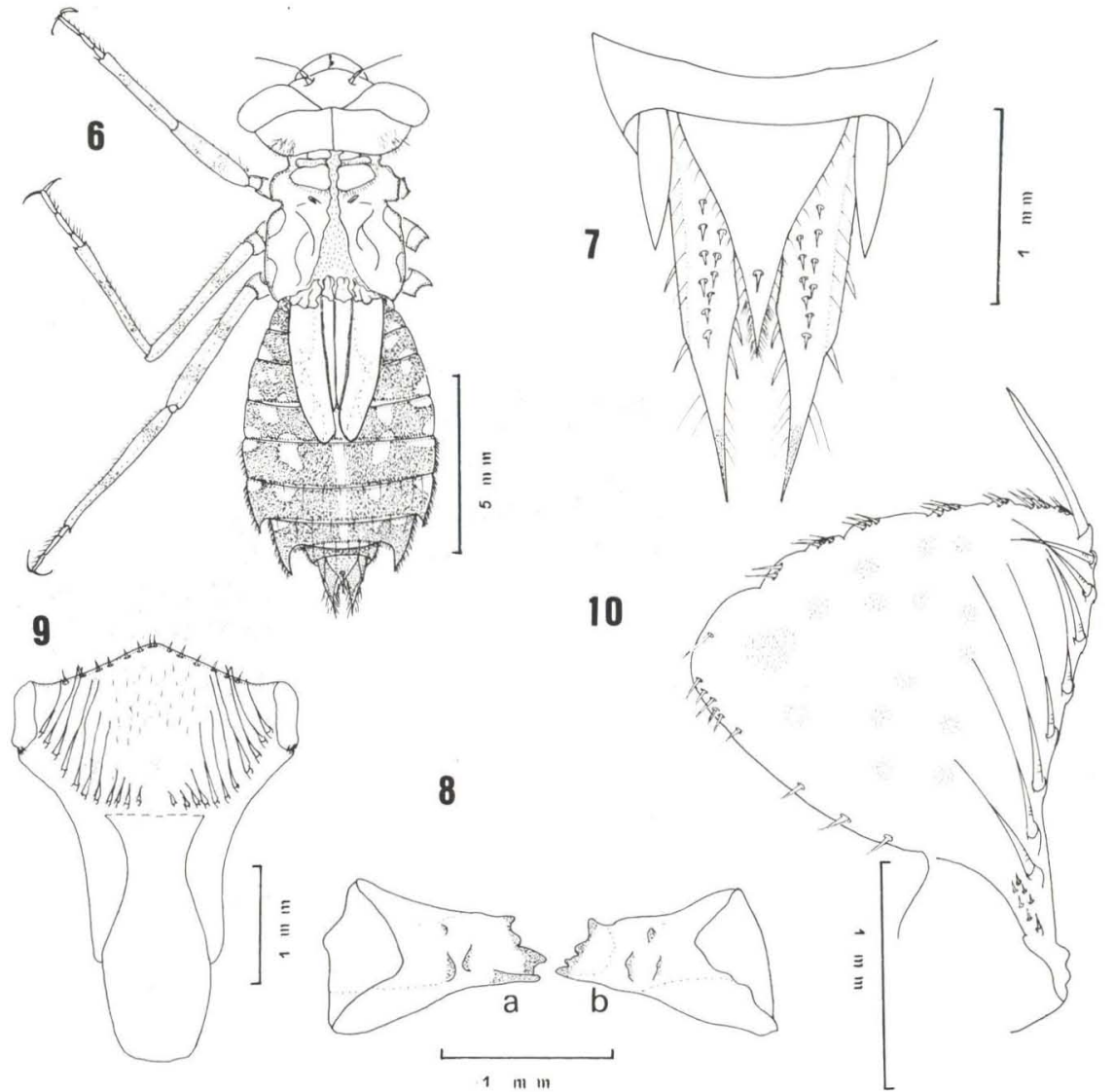

Figs 6-10. Micrathyria hesperis. (6) Exúvia de último instar; (7) apêndiès abdominais; (8) mandíbulas (a - esquerda, b - direita); (9) pré-mento; (10) palpo labial direito.

pequenas cerdas transparentes; dois pequenos e robustos espinhos em cada lado da margem lateral, na conexão com os palpos labiais. Palpo labial largo, com pequenas máculas castanho-escuras; oito setas e uma setela em cada palpo, além da garra móvel; parte basal com seis pequenas setas espiniformes; margem distal com sete crenulações suaves, cada uma suportando de dois a três espinhos crenulares; número de espinhos em cada crenulação, partindo da garra móvel: 2, 3, 2, 3, 3, 3, 2; margem interna com 13 setas irregularmente distribuídas; margem externa lisa.

Protórax com disco dorsal bordeado por cerdas. Tecas alares alcançando a margem posterior do VI segmento abdominal. Pernas longas, armadas de fileiras de espinhos e cerdas; duas bandas escuras evidentes nos fềmures e tíbias dos três pares de pernas.

Abdome com a mesma forma da espécie anteriormente descrita; coloração geral castanha alternando áreas claras com áreas escuras na superfície dorsal e 
dorso-lateral; faixa dorsal clara evidente em toda sua extensão; espinhos laterais presentes nos segmentos VIII e IX; espinho do VIII segmento reto e estendendo-se até $2 / 3$ do comprimento médio-dorsal do IX segmento; espinho do IX segmento levemente curvado e pouco mais de uma vez maior que o comprimento médiodorsal do IX segmento, alcançando 2/3 do comprimento do cerco. Epiprocto largo na base e fortemente afilado na extremidade, guarnecido por um espinho disposto no centro do seu terço posterior. Paraprocto aproximadamente uma vez e meia maior que o epiprocto e levemente curvado para baixo distalmente. Cerco alcançando aproximadamente $3 / 4$ do comprimento do epiprocto, levemente curvado para baixo distalmente. Todos providos de espinhos e pêlos nas extremidades.

Material examinado. BRASIL, Rio de Janeiro: Rio de Janeiro (Rio Soledade, Prata), 14.I. 1972 (emergência 15.I.1972), uma fêmea, N.D. Santos \& J. Machado leg.; (Mini-Horto Sylvio Potsch, Colégio Pedro II, São Cristóvão), 22.X.1985 (emergência 5.XI.1985), um macho, 27.I.1986 (emergência 01.II.1986), uma fềmea, S.R. Pujol leg.; (recreio dos Bandeirantes, Bosque da Barra), área aberta, 20.IV.1991, 39 machos e quatro fêmeas, área tabual, 18.V.1991, dois machos, M.F. Napoli \& C.F. Bizerril leg., área aberta 22.VI.1991, dois machos e uma fêmea, M.F. Napoli \& C.F. Bizerril leg. Depositado no MN-UFRJ.

Medidas (em milímetros). Comprimento total 13,05; comprimento da cabeça 2,4; largura máxima da cabeça 4,5; comprimento dos olhos 1,2; largura máxima dos olhos 0,96 ; comprimento das antenas 2,28 ; comprimento relativo dos antenômeros $15,22,37,30,34,45,45$; comprimento do pré-mento 3,52; largura basal 1,12; largura máxima 3,12; comprimento do palpo labial 2,88; teca alar anterior 3,6; teca alar posterior 3,67; F1, F2, F3 (F=fềmur) 2,64, 2,96, 3,84; $\mathrm{T} 1$, T2, T3 ( $\mathrm{T}=$ tíbia) 2,56, 3,2, 3,56; comprimento do abdome 9,3; largura máxima 3,92; comprimento do espinho lateral VIII segmento 0,48 ; largura basal 0,48 ; comprimento do espinho lateral IX segmento 0,8 ; largura basal 0,64 ; epiprocto 0,94 ; paraprocto 1,42 ; cerco 0,71 .

Distribuição. Brasil, Pará: Tucuruí; Piauí: Simplício Mendes, Paulistana; Ceará: Barbalha; Pernambuco: Engenho Muribora, Recife, São Lourenço; Bahia: Salvador, Serrinha, Jacuipe, Coxibamba; Goiás: Campinas, Formosa, Capivari; Minas Gerais: Lagoa Santa, Janauba, Cataguazes, Lagoa do Palmital, Lagoinha, Leolpodina, Aimorés, Viçosa; Espírito Santo: Santa Tereza, Baixo Guandú, mutum Preto, Colatina, Parque Sooretama, Itaguaçú; Rio de Janeiro: Carmo do Sapucaí, Rio Soledade, São Cristóvão, Recreio dos Bandeirantes; São Paulo: Rio Claro, Pirassununga; Paraná: Curitiba, Parque Nacional do Iguaçu; Rio Grande do Sul: Arroio, Contagem.

\section{Micrathyria mengeri Ris, 1919}

Figs 11-15

Larva de tamanho pouco menor que $M$. borgmeieri, de coloração ocrácea.

Cabeça similar às das espécies anteriormente descritas; fórmula mandibular $L 12340$ abd $\bar{R} \overline{1234} \bar{y} \overline{a b c d}$. 


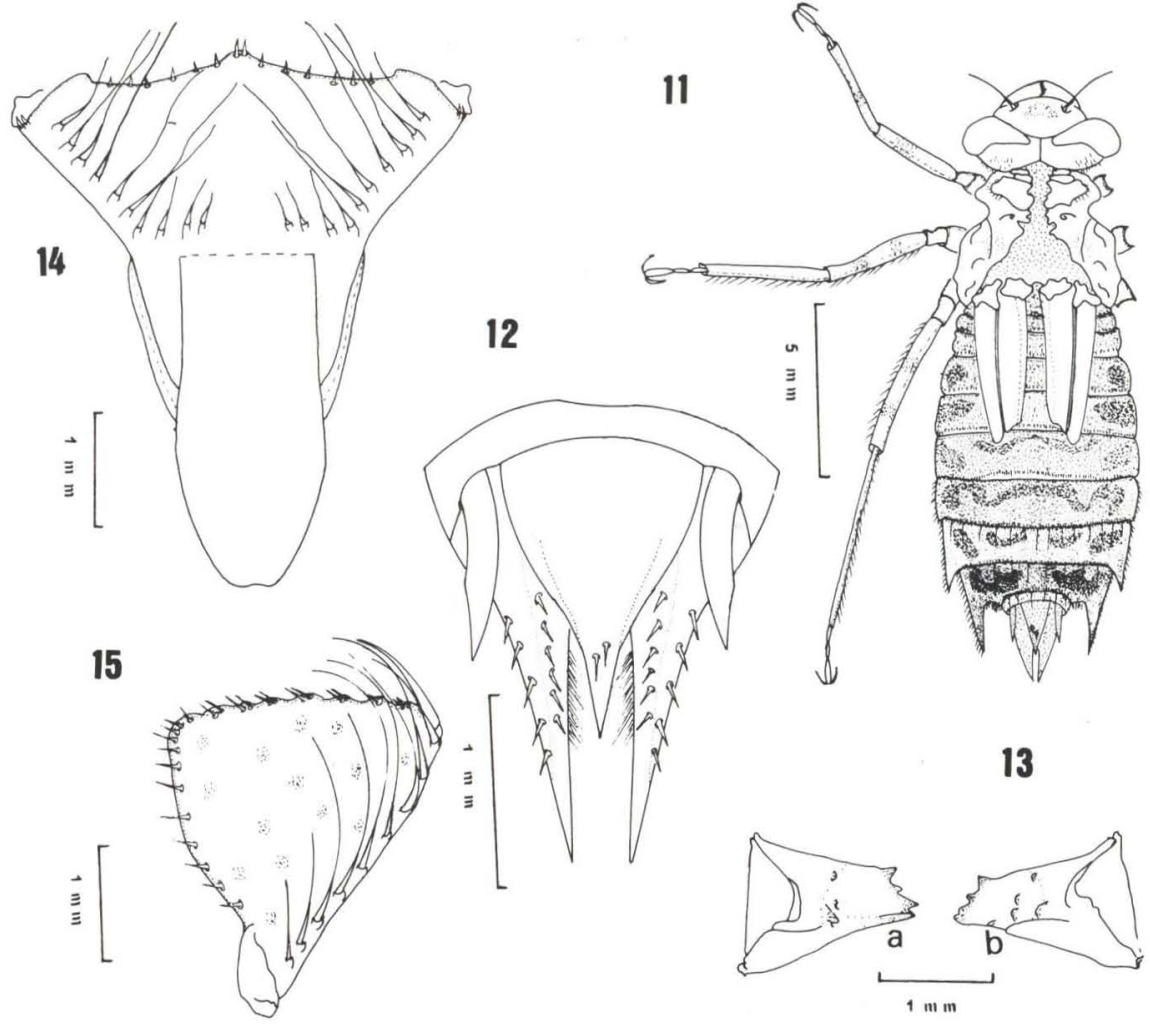

Figs 11-15. Micrathyria mengeri. (11) Exúvia de último ínstar; (12) apêndices abdominais; (13) mandîbulas (a - esquerda, b - direita); (14) pré-mento; (15) palpo labial direito.

Lábio largo, quando dobrado, alcançando o nível do segundo par de pernas. Pré-mento com um par de 10 setas pré-mentais; margem distal com seis setas espiniformes de cada lado e duas apicais; dois pequenos e robustos espinhos de cada lado da margem lateral, na conexão com os palpos labiais. Palpo labial largo, com pequenas máculas castanho-escuras; nove setas e uma setela em cada palpo, além da garra móvel; margem distal com sete crenulações suaves, cada uma suportando de um a dois espinhos crenulares; número de espinhos em cada crenulação, partindo da garra móvel: 2, 1, 2, 2, 2, 2, 2; margem interna com 11 setas irregularmente distribuídas, margem externa lisa.

Protórax com disco dorsal bordeado por cerdas. Tecas alares ultrapassando a margem anterior do VI segmento abdominal. Pernas longas, armadas de fileiras de espinhos e cerdas; uma banda escura evidente nos fêmures e tíbias anteriores e duas nos fêmures posteriores.

Abdome com a mesma forma das espécies anteriormente descritas; coloração geral ocrácea alternando áreas claras com áreas escuras na superfície dorsal e dorso-lateral; espinhos laterais presentes nos segmentos VIII e IX como em $M$. 
borgmeieri. Epiprocto largo na base e fortemente afilado na extremidade; guarnecido por dois espinhos dispostos irregularmente no seu terço posterior. Paraprocto como na espécie anteriormente descrita. Cerco alcançando aproximadamente 2/3 do comprimento do epiprocto, levemente curvado para baixo distalmente. Todos providos de espinhos e pêlos nas extremidades.

Material examinado. BrasiL, Espírito Santo: Goitacases (mata), 22.I.1973 (emergência 28.I.1973), um macho, N.D. Santos leg. Depositado no MN-UFRJ.

Medidas (em milímetros). Comprimento total 16,2; comprimento da cabeça 2,4; largura máxima da cabeça 4,2; comprimento dos olhos 1,12; largura máxima dos olhos 1,76; comprimento das antenas 2,29; comprimento relativo dos antenômeros $18,21,37,30,37,52,34$; comprimento do pré-mento 3,56; largura basal 0,94 ; largura máxima 2,85; comprimento do palpo labial 2,21; teca alar anterior 3,9; teca alar posterior 4,2; F1, F2, F3 (F=fềmur) 2,55, 3,0, 3,75; T1, $\mathrm{T} 2$, T3 ( $\mathrm{T}=$ tíbia) 2,55, 3,6, 4,2; comprimento do abdome 9,45; largura máxima 5,1 ; comprimento do espinho lateral VIII segmento 0,75 ; largura basal 0,3 ; comprimento do espinho lateral IX segmento 1,39; largura basal 0,49; epiprocto 1,42 ; paraprocto 2,21; cerco 0,93.

Distribuição. BRAsIL, Maranhão: Timon; Goiás: Jataí; Minas Gerais: Lagoa Santa; Espírito Santo: Goitacases; Rio de Janeiro: Lagoa de Juturnaiba.

\section{Micrathyria ocellata Martin, 1897}

Figs 16-20

Larva de tamanho pouco menor que $M$. borgmeieri, de coloração ocrácea.

Cabeça similar às das espécies anteriormente descritas; fórmula mandibular $L 12340$ abd $\bar{R} \overline{1234} \bar{y} \overline{a b c d}$.

Lábio largo, quando dobrado, alcançando o nível do segundo par de pernas. Pré-mento com um par de 12 setas pré-mentais; margem distal com cinco setas espiniformes de cada lado e duas apicais; três pequenos e robustos espinhos em cada lado da margem lateral, na conexão com os palpos labiais. Palpo labial largo, com máculas castanho-escuras próximo à margem distal; 10 setas e uma setela em cada palpo, além da garra móvel; margem distal com oito crenulações suaves, cada uma suportando de um a dois espinhos crenulares; número de espinhos em cada crenulação, partindo da garra móvel: $1,1,2,2,1,1,1,1$; margem interna com 12 setas irregularmente distribuídas; margem externa lisa.

Protórax com disco dorsal bordeado por cerdas. Tecas alares alcançando a margem posterior do V segmento abdominal. Pernas longas, armadas de fileiras de espinhos e cerdas.

Abdome com a mesma forma das espécies anteriormente descritas; coloração geral ocrácea alternando áreas claras com áreas escuras na superfície dorsal e dorso-lateral; espinhos laterais presentes nos segmentos VIII e IX; espinho do VIII segmento reto e estendendo-se até $2 / 3$ do comprimento médio-dorsal do IX segmento; espinho do IX segmento levemente curvado e pouco mais de uma vez 
maior que o comprimento médio-dorsal do IX segmento, alcançando 1/3 do comprimento do cerco. Epiprocto largo na base e fortemente afilado na extremidade; guarnecido por dois espinhos dispostos irregularmente no seu terço posterior. Paraprocto como em $M$. hesperis. Cerco alcançando aproximadamente a metade do comprimento do epiprocto, levemente curvado para baixo distalmente. Todos providos de espinhos e pêlos nas extremidades.
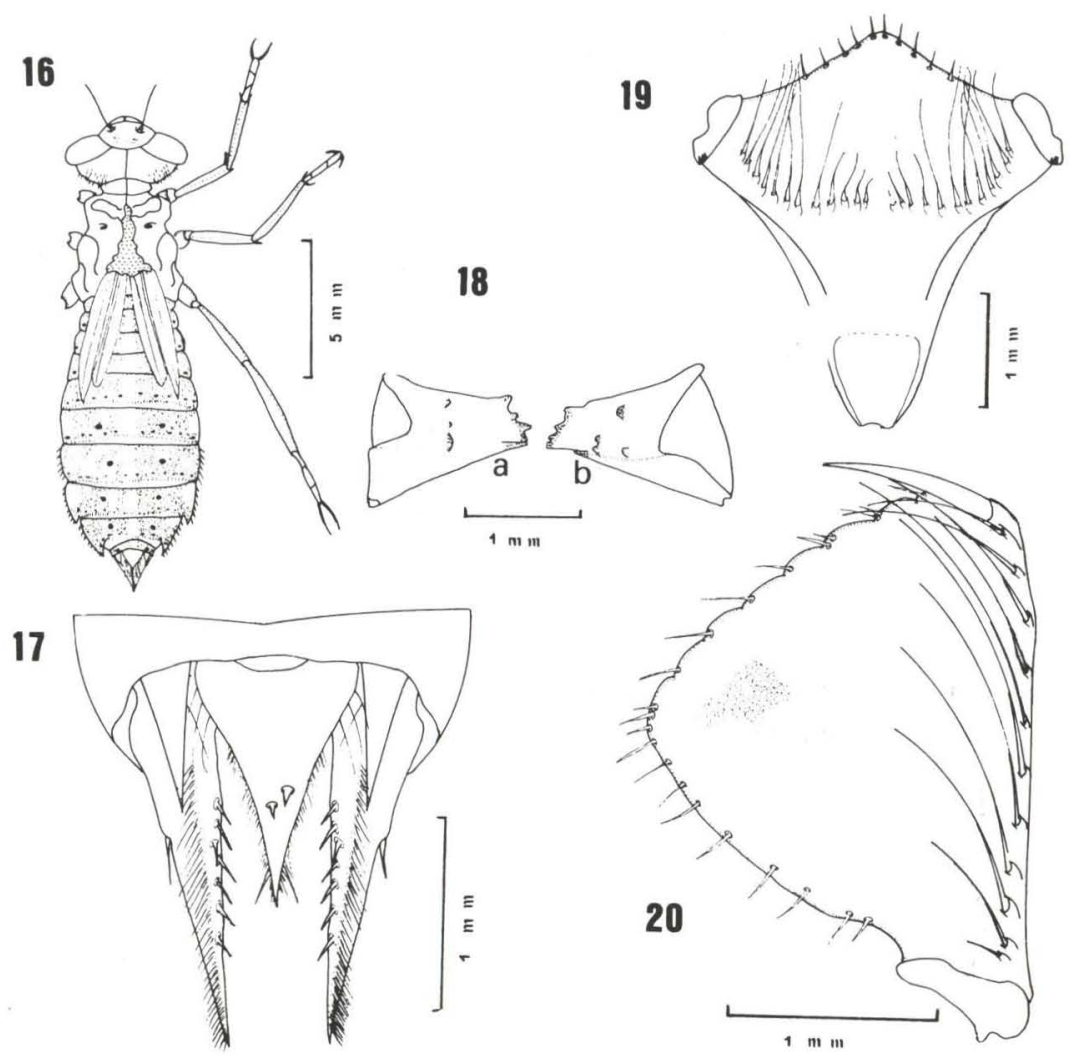

Figs 16-20. Micrathyria ocellata. (16) Exúvia de último instar; (17) apêndices abdominais; (18) mandibulas (a - esquerda, b - direita); (19) pré-mento; (20) palpo labial direito.

Material examinado. BRASIL, Rio de Janeiro: Rio de Janeiro (Barra da Tijuca, Bosque da Barra), área de tabual, 9.I.1991, dois machos, M.F. Napoli \& C.F. Bazerril leg., área aberta, 18.V.1991, 24 machos, M.F. Napoli \& C.F. Bezerril leg., área de tabual, 18.V.1991, dois machos, M.F. Napoli \& C.F. Bezerril leg., área aberta, 22.VI.1991, cinco machos, M.F. Napoli \& C.F. Bezerril leg.; Itaguaí (Campus da Universidade Federal Rural do Rio de Janeiro), 05.V.1993 (emergência 28.V.1993), dois machos, C. A. Cruz leg. Depositado no MN-UFRJ. 
Medidas (em milímetros). Comprimento total 19,0; comprimento da cabeça 2,3; largura máxima da cabeça 4,7; comprimento dos olhos 1,6; largura máxima dos olhos 2,1 ; comprimento das antenas 2,48 ; comprimento relativo dos antenômeros 21, 21, 21, 17, 21, 11, 10; comprimento do pré-mento 3,6; largura basal 1,12; largura máxima 3,12; comprimento do palpo labial 2,36; teca alar anterior 4,2; teca alar posterior 4,8; F1, F2, F3 (F=fềmur) 3,15, 3,6, 3,9; T1, T2, T3 ( $\mathrm{T}=\mathrm{t}$ íbia) $2,4,2,85,2,85$; comprimento do abdome 11,55; largura máxima 5,55; comprimento do espinho lateral VIII segmento 0,44 ; largura basal 0,27 ; comprimento do espinho lateral IX segmento 0,76 ; largura basal 0,36 ; epiprocto 0,98 ; paraprocto 1,46 ; cerco 0,73 .

Distribuição. Brasıl, Acre: Rodrigues Alves; Pará: Belém; Maranhão: Posto Gonçalves Dias; Ceará: ?; Pernembuco: Recife; Bahia: Riachão do Jacuipe, Salvador, Serrinha, Caravelas, Periperi, Represa da Bolandeira; Mato Grosso: Bodoquena; Minas Gerais: Lagoa Santa, Pirapora, Raul Soares, Janaúba; Espírito Santo: Baixo Guandú, Santa Tereza, Conceição da Barra; Rio de Janeiro: Araruama, Pedra de Itaúna, Restinga de Itapeba, Itaguaí, Recreio dos Bandeirantes; São Paulo: Rio Claro, Pirassununga; Paraná: Curitiba; Santa Catarina: Blumenau.

\section{Micrathyria pirassunungae Santos, 1953}

Figs 21-25

Larva de tamanho pouco maior que as precedentes, de coloração geral ocrácea.

Cabeça similar às das espécies anteriormente descritas; fórmula mandibular $L 12340 \quad a b$ $R \overline{1234} \overline{x y} \overline{a b d}$.

Lábio largo, qunado dobrado, alcançando o nível do segundo par de pernas. Pré-mento com um par de 13 setas pré-mentais; margem distal com 10 setas espiniformes de cada lado e duas apicais; superfície interna com aproximadamente 30 pequenas cerdas transparentes; dois pequenos e robustos espinhos em cada lado da margem lateral, na conexão com os palpos labiais; nove setas e uma setela em cada palpo, além da garra móvel; parte basal com 13 pequenas setas espiniformes; margem distal com oito crenulações suaves, cada uma suportando de dois a cinco espinhos crenulares; número de espinhos em cada crenulação, partindo da garra móvel: 2, 2, 3, 4, 3, 5, 5, 5; margem interna com 10 setas irregularmente distribuídas; margem externa lisa.

Protórax com disco dorsal bordeado por cerdas. Tecas alares ultrapssando a margem anterior do VI segmento abdominal. Pernas longas, armadas de fileiras de espinhos e cerdas; duas bandas escuras evidentes nos fêmures e tíbias dos três pares de pernas.

Abdome com a mesma forma das espécies anteriormente descritas; coloração uniforme com manchas escuras dispersas pelos últimos segmentos; espinhos laterais presentes nos segmentos VIII e IX como em $M$. borgmeieri e $M$. mengeri. 
Epiprocto largo na base e fortemente afilado na extremidade, alcançando 3/4 do comprimento do paraprocto. Paraprocto como nas espécies anteriormente descritas. Cerco alcançando aproximadamente $3 / 4$ do comprimento do epiprocto, levemente curvado para baixo distalmente. Todos providos de espinhos e pêlos nas extremidades.
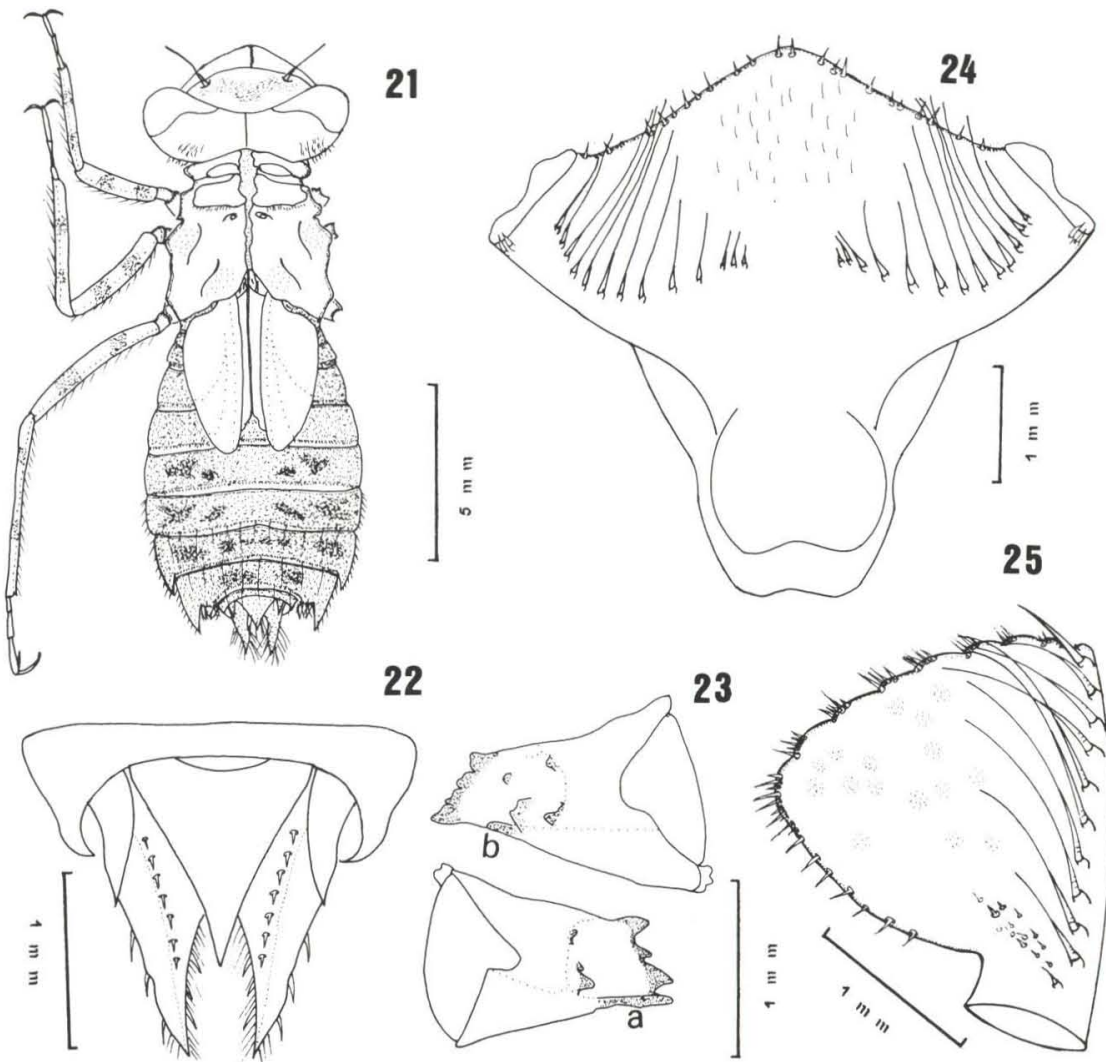

22

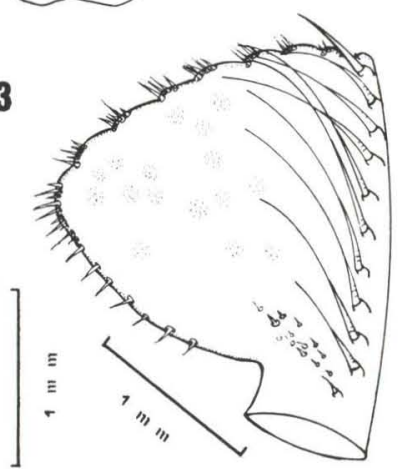

Figs 21-25. Micrathyria pirassunungae. (21) Exúvia de último ínstar; (22) apêndices abdominais; (23) mandibulas (a - esquerda, b - direita); (24) pré-mento; (25) palpo labial direito.

Material examinado. Brasil, Rio de Janeiro: Rio de Janeiro (Fazenda São Joaquim - areal), 10.VII.1969 (emergência 25.VIII.1969), dois machos, N.D. Santos \& J. Machado leg. Depositado no MN-UFRJ.

Medidas (em milímetros). Comprimento total 17,1; comprimento da cabeça 2,85; largura máxima da cabeça 4,65; comprimento dos olhos 1,52; largura máxima dos olhos 1,6; comprimento das antenas 2,96; comprimento relativo dos antenômeros $26,30,52,41,46,49,52$; comprimento do pré-mento 4,5; largura basal 1,8; largura máxima 4,2; comprimento do palpo labial 2,85; teca alar anterior 5,12; teca alar posterior 5,2; F1, F2, F3 (F=fêmur) 3,12, 3,68, 4,64; T1, T2, 
$\mathrm{T} 3$ ( $\mathrm{T}=$ tíbia) 3,6, 4,32, 5,36; comprimento do abdome 9,6; largura máxima 5,7: comprimento do espinho lateral VIII segmento 0,64 ; largura basal 0,32 ; comprimento do espinho lateral IX segmento 0,8 ; largura basal 0,4 ; epiprocto 0,94 ; paraprocto 1,35 ; cerco 0,67 .

Distribuição. Brasil, Mato Grosso: Chapada dos Guimarães, Alto das Garças; Goiás: Formosa, Jataí, Cachoeira Alta; Distrito Federal: Brasília (Reserva IBGE, Planaltina); Minas Gerais: Tupaciguara, Lagoa Santa, São João Del Rei, Caraça, Estrada Belo Horizonte-Brasília; Espírito Santo: Santa Tereza; Rio de Janeiro: Rio de Janeiro (Fazenda São Joaquim); São Paulo: Pirassununga.

\section{Micrathyria stawiarskii Santos, 1953}

Figs 26-30

Larva de tamanho pouco maior que as precedentes, de coloração ocrácea.

Cabeça similar às das espécies anteriormente descritas; fórmula mandibular $L 12340 \quad a b$ $R 1234$ xy ahd.

Lábio largo, quando dohrado, alcançando o nível do segundo par de pernas. Pré-mento com um par de 12 setas pré-mentais; margem distal com sete setas espiniformes de cada lado e três apicais; superfície interna com aproximadamente 41 pequenas cerdas transparentes; três pequenos e robustos espinhos de cada iado da margem lateral, na conexão com os palpos labiais. Palpo labial largo, com pequenas máculas castanho-escuras; nove setas e uma setela em cada palpo, além da garra móvel; parte hasal com 13 pequenas setas espiniformes; margem distal com oito crenulações suaves, cada uma suportando de três a cinco espinhos crenulares; número de espinhos em cada crenulação, partindo da garra móvel: 3 . $3,4,5,4,4,5,4$; margem interna com sete setas irregularmente distribuídas: margem externa lisa.

Protórax com disco dorsal bordeado por cerda. Tecas alares alcançando a margem anterior do $\mathrm{V}$ segmento ahdominal. Pernas longas, armadas de fileiras de espinhos e cerdas.

Ahdome com a mesma forma das espécies anteriormente descritas; coloração uniforme; espinhos laterais presentes nos segmentos VIII e IX como em $M$. hesperis. Epiprocto largo na base e fortemente atilado na extremidade: alcançando 2/3 do comprimento do paraprocto; guarnecido por um espinho disposto no centro de seu terço posterior. Paraprocto aproximadamente uma vez e meia maior que o epiprocto e levemente curvado para haixo distalmente. Cerco alcançando aproximadamente 2/3 do comprimento do epiprocto, levemente curvado para baixo distalmente. Todos providos de espinhos e pêlos nas extremidades.

Material examinado. BRAsil, Minas Gerais: São João Del Rei (Serra dos Lenheiros), 11.II. 1974 (emergência 14.II.1974), um macho, N.D. Santos leg.; São Lourenço, 20.I.1976 (emergência 5.II.1976), um macho, N.D. Santos \& Duilio leg.. Rio de Janeiro: Rio de Janeiro (Recreio dos Bandeirantes, Bosque da Barra), área de tahual, 20.IV.1991, dois machos, área de tahual, 18.V.1991, três 

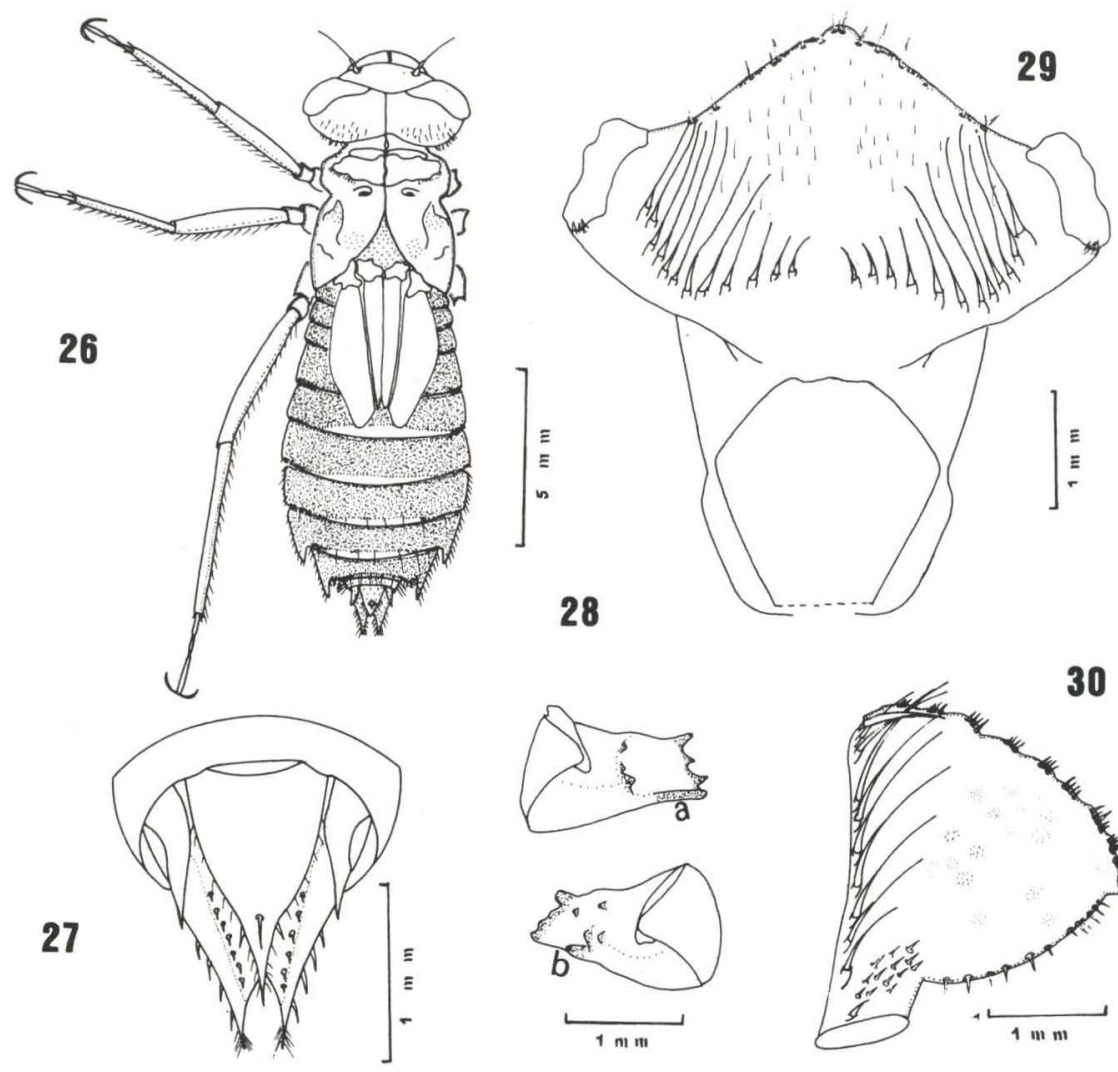

Figs 26-30. Micrathyria stawiarskii. (26) Exúvia de último ínstar; (27) apêndices abdominais; (28) mandibulas (a - esquerda, b - direita); (29) pré-mento; (30) palpo labial direito.

machos e uma fềmea, M.F. Napoli \& C.F. Bezerril leg. Depositado no MN-UFRJ.

Medidas (em milímetros). Comprimento total 18,9; comprimento da cabeça 3,3; largura máxima da cabeça 4,95; comprimento dos olhos 1,36; largura máxima dos olhos 1,68; comprimento das antenas 3,0 ; comprimento relativo dos antenômeros $26,34,53,37,42,52,56$; comprimento do pré-mento 5,2; largura basal 1,36; largura máxima 4,72; comprimento do palpo labial 2,96; teca alar anterior 5,36; teca alar posterior 5,6; F1, F2, F3 ( F=fêmur) 3,2, 4,08, 4,96; T1, T2, T3 ( $\mathrm{T}=$ tíbia) 3,92, 4,0, 5,52; comprimento do abdome 9,3; largura máxima 6,0; comprimento do espinho lateral VIII segmento 0,84 ; largura basal 0,30 ; comprimento do espinho lateral IX segmento 1,05; largura basal 0,46; epiprocto 1,06; paraprocto 1,48 ; cerco 0,78 .

Distribuição. BRASIL, Goiás: Goiânia, Formosa; Distrito Federal: Brasília, Rio Bananal, Rio Sobradinho, Corredeira Veredinha, Reserva IBGE, Ituituba; Minas Gerais: São Louranço, São João Del Rei, Serra do Cipó, Lagoa Santa, 
Lagoa Dourada, Lagoa do Palmital, Jaboticatubas, Carmo do Rio Claro, ConseIheiro Lafaiete, Caxambu, Viçosa, Barbacena, Gouveia, Caraça; Rio de Janeiro: Rio de Janeiro, Teresópolis, Friburgo, Areal, Itatiaia, Serra da Bocaina, Vassouras, Carmo do Sapucaí; Sào Paulo: Boracéia, Larvinha; Paraná: Curitiba; Santa Catarina: Ponte Alta, Blumenau, Lages; rio Grande do Sul: Alegrete, Rosário, Jaguarão, Parque Nacional Aparados da Serra, Lajeado Grande, Santo Augusto.

\section{DISCUSSÃO E CONCLUSÕES}

Foram realizadas sete coletas em ambientes lênticos temporários na Baixada de Jacarepaguá, Rio de Janeiro, no período de janeiro a outubro de 1991, resultando 106 exemplares de quatro espécies distintas. cujas larvas ainda são desconhecidas para a ciência: $M$. horgmeieri, $M$. hesperis, $M$. ocellata e $M$. stawiarskii. Sete outros exemplares de $M$. ocellata foram coletados em ambientes lênticos permanentes, provenientes da área da Universidade Federal Rural do Rio de Janeiro, Itaguaí, Rio de Janeiro.

As larvas das diferentes espécies foram criadas em lahoratório, em pequenas caixas de isopor e alimentadas com larvas de mosquito. Do total de larvas criadas das diferentes localidades, somente a de $M$. ocellata sofreu eclosão total. A identificação das outras cinco espécies foi possível através da comparação com o material eclodido e identificado já existente na Coleção do Museu Nacional.

A distribuição das espécies no Brasil foi haseada no material existente na Coleção do Museu Nacional (adultos e larvas) (Fig. 31).

Das espécies de Micrathyria coletadas em ambientes naturais temporários, ohservou-se haver uma predominância de $M$. hexperis e $M$. hypodidyma em relação as outras espécies. Em menor quantidade foram coletadas $M$. atra, $M$. borgmeieri e $M$. stawiarskii. As larvas das diferentes espécies são identificáveis através da combinação dos seģuintes caracteres: fórmula mandibular, número de setas pré-mentais, número de setas palpais e número de espinhos no epiprocto. Entretanto, M. mengeri e $M$. ocellata apresentam o mesmo padrão de fórmula mandibular, o mesmo ocorrendo para $M$. pirassunungae e $M$. stawiarskii.

A avaliação das fórmulas mandibulares de $M$. artemis, $M$. atra e $M$. hypodidyma apresentaram os seguintes padrões: $M$. artemis e $M$. atra L 12340 abc $R 1234$ y abd e $M$. hypodidyma $R 1234$ y abd

Não foi possivel analisar a fórmula mandibular de $M$. didyma, pois na descrição da larva NEEDHAM (1943) não cita e não apresenta esquema da mandîhula e não possuímos exemplares imaturos desta espécie na Coleção.

A fauna odonatológica associada foi representada pelas seguintes espécies: Tramea cophysa Hagen, 1867; Erythrodiplax sp. e Micrathyria atra, entre os Libellulidae; Anax concolor Brauer, 1865 e Coryphaeshna perrensi McLachlan, 1887, entre os Aeshnidae; Ischmura (Ceratura) capreolus (Hagen, 1861) entre os Coenagrionidae e Lestex hipupillatus Calvert, 1909 e Lestes tricolor Erichson, 1848 entre os Lestidae. 


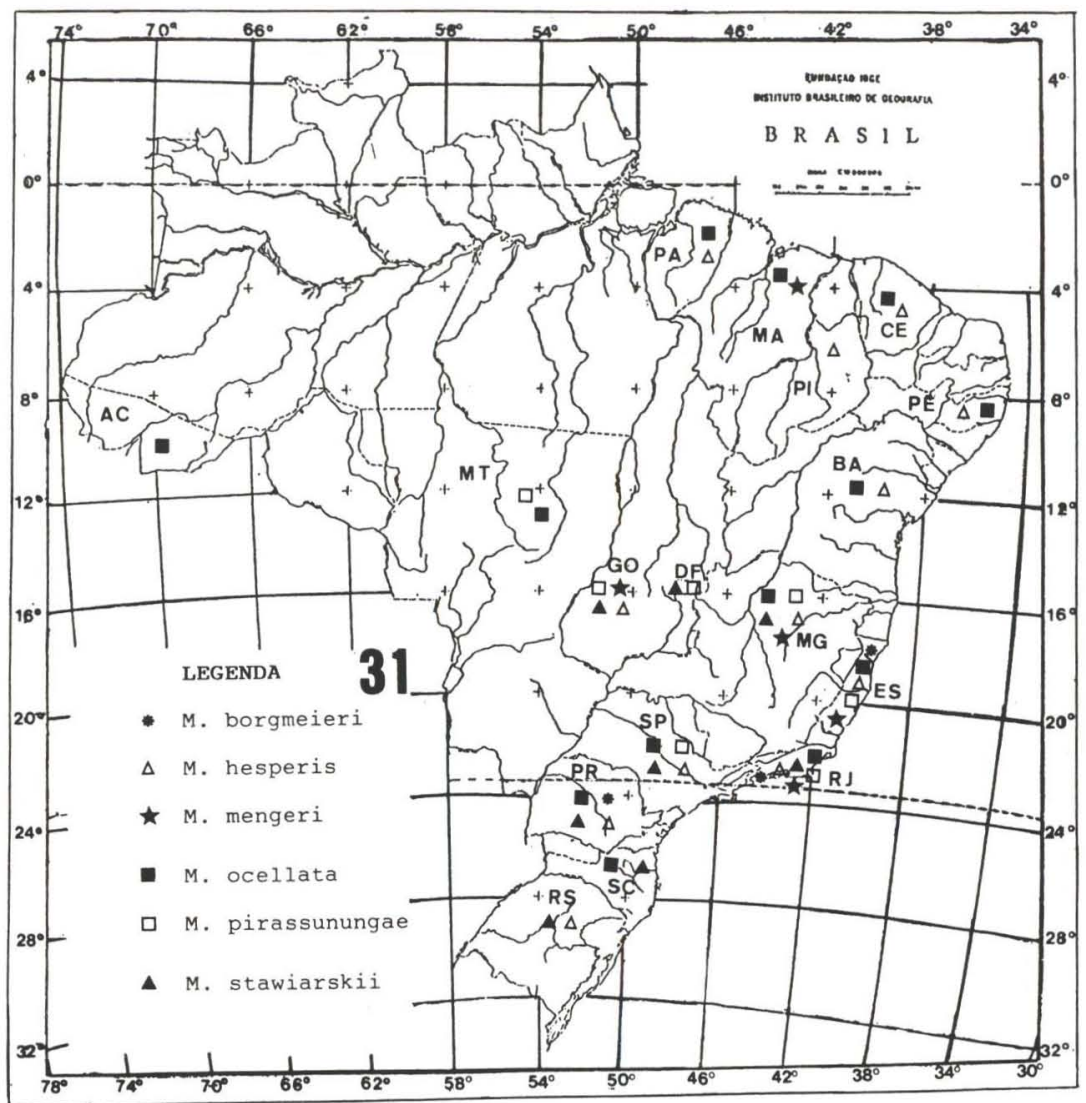

Fig. 31. Distribuição geográfica de $M$. borgmeieri, $M$. hesperis, $M$. mengeri, $M$. ocellata, $M$. pirassunungae e $M$. stawiarskii no Brasil. Acre (AC), Bahia (BA), Ceará (CE), Distrito Federal Brasilia (DF), Espírito Santo (ES), Goiás (GO), Maranhão (MA), Mato Grosso (MT), Minas Gerais (MG), Pará (PA), Paraná (PR), Pernambuco (PE), Piauí (PI), Rio Grande do Sul (RS), Rio de Janeiro (RJ), Santa Catarina (SC), São Paulo (SP),

Agradecimentos. Agradecemos aos Professores José Roherto Pujol-Luz, da Universidade Federal Rural do Rio de Janeiro e Alcimar do Lago Carvalho, da Universidade Federal do Rio de Janeiro pelas valiosas sugestões.

\section{REFERÊNCIAS BIBLIOGRÁFICAS}

DAviES, D.A.L. \& P. ToBIN. 1985. The dragonflies of the world: a systematic list of the extant species of Odonata. Vol. 2 Anisoptera. Soc. Int. Odonatol. 5 (supl.): 1-151.

Needham, J.G. 1943. Life history notes on Micrathyria. Ann. Ent. Soc. Amer. 36 (2): 185-189. 
Rodrigues Capitulo, A. 1988. Micrathyria ringueleti Spec. Nov., a new dragonfly from Argentina and its larva (Anisoptera: Libellulidae). Odonatologica 17 (4): 409-48.

SanTOS, N.D. 1968. Contribuição conhecimento da fauna do Estado da Guanabara. 62. Notas sobre a ninfa e o imago de Micrathyria hypodidyma Calvert, 1906. Atas Soc. Biol. Rio de Jan. 11 (5): 195-197.

- 1972. Contribuição ao conhecimento da fauna do Estado da Guanabara e arredores. 80. Descrição da ninfa de Micrathyria artemis (Selys, ms) Ris, 1911 (Odonata: Libellulidae). Atas Soc. Biol. Rio de Jan. 15 (3): 141-143.

- 1978. Contribuição ao conhecimento da fauna do Município do Rio de Janeiro, RJ e arredores. 85. Descrição da ninfá de Micrathyria atra (Martin, 1897) Calvert, 1906 (Odonata: Libellulidae). Atas Soc. Biol. Rio de Jan. 19: 17-18.

1988. Catálogo bibliográfico de ninfas de odonatos neotropicais. Acta Ámazonica 18 (1-2): 265-350.

SANTOS, N.D. \& A.B. MACHADO. 1983. New records of dragonflies for the state of Minas Gerais, Brazil. Notul. Odonatol. 2 (2): 17-32.

SANTOS, N.D.; J.M. COSTA \& J.R. PUJOL-LUZ. 1988. Nota sobra a ocorrência de odonatos em tanques de piscicultura e o problema da predação de alevinos pelas larvas. Ata Limnol. Brasil. 2: 771-780.

WATSON, M.C. 1956. The utilization of mandibular armature in taxonomic studies of anisopterous nynphs. Trans. Am. Ent. Soc. 81: 155-205.

Westfall, M.J. 1992. Notes on Micrathyria, with descriptions of $M$. pseudoeximia sp.n., $M$. occipita sp.n., $M$. dunklei sp.n. and $M$. divergens sp.n. (Anisoptera: Libellulidae). Odonalogica 21 (2): 203-218. 